\title{
Balkanologie
}

Balkanologie Revue d'études pluridisciplinaires

Vol. VII, $n^{\circ} 1$ | 2003

Volume VII Numéro 1

\section{Mythes et réalités de la convergence russo- yougoslave pendant la crise ou Kosovo}

Jean-Robert Raviot

\section{(2) OpenEdition}

12 Journals

Édition électronique

URL : http://journals.openedition.org/balkanologie/480

DOI : $10.4000 /$ balkanologie.480

ISSN : 1965-0582

Éditeur

Association française d'études sur les Balkans (Afebalk)

Édition imprimée

Date de publication : 1 juin 2003

Pagination : 159-169

ISSN : 1279-7952

\section{Référence électronique}

Jean-Robert Raviot, « Mythes et réalités de la convergence russo-yougoslave pendant la crise ou

Kosovo », Balkanologie [En ligne], Vol. VII, n 1 | 2003, mis en ligne le 18 février 2009, consulté le 17 décembre 2020. URL : http://journals.openedition.org/balkanologie/480 ; DOI : https://doi.org/

10.4000/balkanologie.480 


\title{
MYTHES ET RÉALITÉS DE LA CONVERGENCE RUSSO-YOUGOSLAVE PENDANT LA CRISE DU KOSOVO
}

\author{
Jean-Robert Raviot*
}

Au printemps 1999, l'intervention des forces armées de l'OTAN en Yougoslavie est vivement condamnée par la Russie. L'argumentation de Moscou est fondée sur un implacable argument de droit : conformément à la Charte des Nations Unies, l'intégrité territoriale des États souverains qui constituent la communauté internationale ne saurait être remise en cause, fût-ce au nom du droit d'ingérence, que par un vote du Conseil de Sécurité de l'ONU. Le ministre russe des Affaires étrangères, Igor Ivanov, résume en ces termes la position officielle russe sur l'intervention des puissances occidentales : "Menée au nom de prétendus idéaux démocratiques, l'intervention de l'OTAN en RFY constitue une agression contre un Etat souverain, un blasphème contre ces mêmes idéaux (...) qui a déclenché la catastrophe humanitaire qu'elle prétendait vouloir prévenir $n^{1}$.

Le 24 mars 1999, premier jour des frappes aériennes de l'Alliance atlantique contre la Yougoslavie, le Premier ministre russe, Evgueni Primakov, s'envolant vers Washington, fait demi-tour au-dessus de l'Océan atlantique. Voilà qui symbolise très médiatiquement le bref épisode de nouvelle guerre froide entre la Russie et l'Occident qui commence alors. Les grands médias, occidentaux comme russes, soulignent la convergence de Moscou et de Belgrade, les premiers pour jeter le discrédit sur le fondement réel de la position russe - respect scrupuleux du droit international ou vieilles connivences aux relents panslavistes et communistes ? -, les seconds pour donner un supplément d'âme à une alliance que l'on sait de pure circonstance. Tout viendrait concourir à rapprocher les deux pays, deux États successeurs en titre de fédérations socialistes 
démantelées, deux pays slaves orthodoxes historiquement alliés, deux États aujourd'hui fragilisés aux prises avec une minorité nationale de confession musulmane - les Albanais du Kosovo et les Tchétchènes - dont l'insurrection remet en cause leur intégrité territoriale ${ }^{2}$. Deux perdants de l'Histoire, ironisent les uns; deux victimes des manigances occidentales, assènent les autres.

Quelle est la réalité de cette convergence russo-yougoslave ? Il faut évaluer le poids des réseaux russo-yougoslaves et, plus généralement, de ceux qui se font le relais de l'influence russe en Yougoslavie (RFY). Pour ce faire, il s'agit d'abord de repérer ces réseaux d'influence (influence sous toutes ses formes: économique, politique, culturelle, militaire, ...). Nous nous en tiendrons ici à cette première étape. Au printemps 1999, pour la première fois dans l'histoire de ce théâtre stratégique post-yougoslave (entendu comme le territoire de l'ancienne RSFY) investi depuis six ans par les grandes puissances, un État - et non plus seulement des entités autoproclamées ou des réseaux officieux, voire occultes - est clairement désigné comme ennemi. Les puissances occidentales ne prônent plus l'interposition entre des belligérants, mais l'intervention militaire pour soutenir un allié face à un adversaire plus fort, personnifié par le président yougoslave, Slobodan Milošević.

Nous allons tenter de repérer les points de convergence russo-yougoslaves et d'identifier quelques-uns de ses acteurs pendant la crise du Kosovo.

1) La crise du Kosovo est d'abord un jeu diplomatique qui s'installe entre les grandes puissances, avec ceci de particulier que l'une d'entre elles - les États-Unis - mène le jeu, les autres (Grande-Bretagne, France, Allemagne, Russie, Chine) devant se positionner le plus favorablement possible par rapport à elle, ce qui entraîne une sorte de compétition entre ces dernières. L'intervention de l'OTAN conduit la diplomatie russe à adopter une tactique dont on retracera brièvement le déroulement et dont on essaiera ensuite d'évaluer les objectifs et les limites.

2) La crise du Kosovo libère un déferlement de passions qui mobilisent bien des troupes : les armées régulières et les mercenaires qui combattent aux côtés des milices albanaises ou serbes ainsi que les cohortes de journalistes, d'analystes, d'experts et d'intellectuels qui fournissent aux médias les interprétations et les cadres d'analyse qui façonnent l'espace public. Les passions stimulent la construction d'appareillages idéologiques rivaux fabriqués par les appareils (États en présence, OTAN). Parmi ces appareillages, le néo-panslavisme expliquerait la solidarité ("atavique" 3 , pour les uns) des Russes avec leurs "frères serbes", slaves et chrétiens. Manipulé par les médias et utilisé en avril-mai 1999 par

\footnotetext{
${ }^{2}$ Rapprochement qui expliquerait une perception différente de la crise du Kosovo par l'opinion publique russe selon Serebrjannikov (V.), " Kosovskaja i cecenskaja vojny v massovom soznanii Rossii i zapada ", Sociologiceskie issledovanija, (9), 2000.
}

3 Cf. l'éditorial du quotidien Le Monde, 14/06/99. 
la classe politique et, dans une bien moindre mesure, par la diplomatie russes, le néo-panslavisme est retourné contre la Russie par des experts et responsables occidentaux dans une amorce de campagne anti-russe en juin 1999.

3) La crise du Kosovo constitue enfin un terrain propice au déploiement de stratégies d'acteurs (économiques) dont les desseins ne sont pas proprement politiques mais qui jouent de leur influence pour orienter le règlement du conflit, en servant par exemple de relais d'influence. C'est à ce niveau que la réalité pragmatique des rapports russo-yougoslaves apparaît au grand jour : les fantasmes panslaves ne sont qu'un écran de fumée. Les relations russo-yougoslaves sont gouvernées par les hydrocarbures (le pétrole et surtout le gaz), elles sont construites par et pour les agents de ces secteurs économiques qui exercent une influence prépondérante sur la diplomatie. On assiste ici à une "oligarchisation" (montée en puissance des oligarques, grands patrons des holdings) de la diplomatie russe, tendance repérable sur d'autres terrains européens, les intérêts économiques devenant prépondérants au fur et à mesure que les bombardements de l'OTAN sur la Yougoslavie prennent de l'ampleur et que, par conséquent, le marché de la reconstruction devient attractif.

\section{LE JEU DIPLOMATIQUE RUSSE}

Vue de Moscou, la crise du Kosovo 4 constitue le point d'orgue d'une tension avec les pays occidentaux, tension dont l'escalade commence en 1997 lorsque, lors du sommet de l'OTAN qui se tient à Madrid, l'Alliance atlantique accepte l'intégration de nouveaux États membres frontaliers de la Russie, notamment les trois États baltes. Depuis lors, Evgueni Primakov, d'abord en tant que ministre des Affaires étrangères (de 1994 à 1998), ne perd pas une occasion de manifester la vive opposition de son pays à l'élargissement de l'OTAN et de rappeler la vocation de la Russie à devenir l'un des pôles de ce "monde multipolaire" qu'il appelle de ses voeux. Cette rhétorique est singulièrement atténuée après que la crise financière qui frappe la Russie en août 1998 oblige les dirigeants russes à faire profil bas pour se voir garantir l'aide indispensable du FMI. Dans ce contexte, la rencontre du 26 janvier 1999 entre le secrétaire d'Etat américain Madeleine Albright et Igor Ivanov, au lendemain des massacres de Račak, se solde par une déclaration officielle commune qui enjoint la Yougoslavie de respecter les droits de l'homme. On note que si le communiqué

4 Sur le déroulement de la crise du Kosovo et son règlement, cf. Sagramoso (Domitilla), * Why did Milosevic Give In ? Political Cooperation in Retrospect n, in Spillmann (Kurt), Wenger (Andreas), eds., Studies in Contemporary History and Security Policy, vol.5: Lessons Learned for International Cooperative Security, Berne : Peter Lang, 2000. 
final est consacré pour moitié à la question du Kosovo, aucune question n'est posée à ce sujet au cours de la conférence de presse qui suit la rencontre : le Kosovo est loin de constituer une priorité sur l'agenda russo-américain 5 .

Peu avant le début des frappes aériennes de l'OTAN, Igor Ivanov déclarait que "l'OTAN ne doit en aucun cas se substituer à l'ONU ", mentionnant principalement les craintes de la Russie de voir s'élargir une "OTAN dont on ne sait plus si elle est une organisation défensive ou offensive " ${ }^{6}$. La question du Kosovo était, en tant que telle, à peine mentionnée. Le 24 mars 1999, une conférence de presse du ministre des Affaires étrangères et du chef d'Etat-major donne le ton officiel général des trois mois à venir : " quels sont donc les idéaux que l'on doit impérativement défendre avec des fusées et des bombes ? N'y a-t-il pas blasphème lorsqu'on bombarde une population civile pacifique en prétendant qu'on le fait pour des motifs humanitaires ? "7. Le communiqué qui clôt la rencontre Ivanov-Albright à Oslo, le 13 avril, témoigne de la radicalisation (au moins verbale) de la position russe : « la nature véritable de l'OTAN apparaît au grand jour : c'est une organisation agressive qui foule aux pieds les principes du droit international $n^{8}$. Il est toutefois significatif que l'agresseur est toujours l'OTAN et jamais nommément les États-Unis, alors que le discours tenu par les gouvernants russes - à commencer par le Président Boris Eltsine sur la scène politique interne désigne expressément les États-Unis comme l'agresseur principal. L'opposition rhétorique à l'intervention armée occidentale s'accompagne d'une offensive diplomatique immédiate. Le 14 avril 1999, Viktor Tchernomyrdine, ancien Premier ministre (de 1992 à 1998) et ancien président du monopole gazier russe Gazprom est nommé envoyé spécial du gouvernement russe auprès des autorités yougoslaves. Les liens de Tchernomyrdine avec le lobby gazier constituent sans doute le motif essentiel de sa désignation (cf. supra). Le 6 mai, le ministre des Affaires étrangères et le premier chef-adjoint de l'État-major annoncent la mise en oeuvre une aide humanitaire à la Yougoslavie, présentée comme la première étape d'une opération à venir sous l'égide de l'ONU. L'aide est modeste : elle ne s'élève qu'à 25 millions de roubles (un peu moins d'i million de dollars !) prélevés sur le budget "action internationale" du Ministère des situations d'urgence ${ }^{9}$. Selon l'ancien conseiller du Président Carter, l'influent analyste Zbigniew Brzezinski, cette opération en masque une autre, de nature militaire, qui vise à assurer

5 Diplomaticeskij Vestnik, (1), janvier 1999.

${ }^{6}$ Diplomaticeskij Vestnik, (3), 1999.

7 Ibid.

${ }^{8}$ Diplomaticeskij Vestnik, (4), avril 1999.

9 L'aide comprend principalement le versement d'une assistance financière, la livraison de 100 automobiles, la construction d'un hôpital mobile pour les réfugiés se trouvant en Macédoine. Cf. Diplomaticeskij Vestnik, (5), mai 1999. 
une présence sur le terrain avant les puissances occidentales afin de réaliser " un plan secret russo-yougoslave de démantèlement du Kosovo en plusieurs zones d'influence " destiné à " garantir une zone d'influence majeure pour Belgrade $n^{10}$. Rétrospectivement, cette assertion apparaît comme le prélude avorté de l'une des nombreuses opérations de désinformation qui furent promues au rang d'armes offensives de premier plan au cours des guerres yougoslaves des années $1990^{11}$.

En se démarquant des options de la politique occidentale, les gouvernants russes obéissent autant à de pressants motifs d'ordre interne qu'à une volonté déterminée d'assurer un rôle pour la Russie dans l'après-guerre. Début mai, la Douma a engagé une procédure de destitution du Président dans le contexte de conflits dans le cadre de la succession à la tête du Kremlin. La diplomatie russe cherche à faire valoir ses capacités d'intermédiation avec le pouvoir yougoslave afin d'offrir aux belligérants occidentaux des concessions de Belgrade, voire la reddition de Milošević, et redorer ainsi son blason auprès des chancelleries occidentales. L'objectif de la diplomatie russe est bien de récolter quelques-uns des fruits de l'après-guerre. Dès sa nomination et ce tout au long du mois de mai, Viktor Tchernomyrdine fait preuve de retenue et de modération. Cependant, c'est en vain que Moscou exige une forte présence russe au sein d'un futur contingent de maintien de la paix au Kosovo placé sous mandat de l'ONU et, surtout, que Belgrade puisse garder des troupes au Kosovo. En dépit de l'échec de la Russie à faire valoir ses demandes, c'est son émissaire, Viktor Tchernomyrdine - qui a pris soin d'approuver préalablement tout plan de paix ! - qui représente le G8 à Belgrade afin de convaincre Milošević d'accepter les conditions (occidentales) de paix. Le chef de l'Etat yougoslave se soumet dès le lendemain. La diplomatie russe, qui se présente - à défaut d'en être l'auteur - comme le garant du règlement de paix, fait mine de récolter les fruits de négociations qu'elle n'a pas réellement conduites...

Sur la scène politique interne, et notamment chez les tenants de la "solidarité slave", cette victoire est perçue comme une capitulation supplémentaire des élites au pouvoir devant les exigences américaines ${ }^{12}$. Tous les experts, même les plus proches du pouvoir, soulignent que la "déclaration de victoire cache un échec ", l'objectif annoncé par Igor Ivanov dès le début de la crise ayant toujours été un règlement pacifique ${ }^{13}$. Pour certains observateurs, cet

${ }^{10}$ Cité par Lewis (Flora), " A Clash with Russia in Kosovo Came Too Close for Comfort ", International Herald Tribune, 01/1099.

${ }^{11}$ Sur ce point, Volkoff (Vladimir), Petite histoire de la désinformation, Paris : Rocher, 1999, chap. 16.

${ }^{12}$ Mjalo (Ksenija), Rossija i poslednie vojny XX vexa, Moskva : Vece, 2002, notamment pp. 284-319.

${ }^{13}$ Colin (Guillaume), Actions, discours, opinion : la crise du Kosovo en Russie ou la structuration de la politique étrangère autour d'une crise, DEA Analyse comparative des aires politiques, IEP de Paris (dir. Anne de Tinguy), 2000. 
alignement est un ajustement imposé par les réalités, car la défaillance des moyens militaires russes sur le terrain entravait a priori toute possibilité d'initiative régionale. Les forces russes furent constamment dépendantes de l'OTAN (et le sont encore) pour l'entretien de leurs troupes sur place. Cette subordination est mal vécue par les officiers supérieurs russes sur le terrain. En témoigne le "coup" de l'aéroport de Pristina (le 11 juin 1999) : en faisant entrer 200 parachutistes russes au Kosovo ("Nous étions les premiers à Priština, comme à Berlin ", déclare l'un des chefs militaires russes à la presse) pour prendre le contrôle de l'aéroport, les généraux russes ont cherché à protester contre le manque de moyens mis à leur disposition, ainsi que contre ce qu'ils perçoivent comme l'échec de Tchernomyrdine et " le résultat de la mauvaise coordination entre la diplomatie, l'Etat-major et les troupes sur le terrain ${ }^{14}$.

La politique russe à l'égard de la Yougoslavie est marquée du sceau d'une réelle méconnaissance du pays, de ses institutions et de ses réseaux politiques. La Yougoslavie - comme les autres pays d'Europe balkanique - semble faire l'objet d'un suivi assez superficiel au sein du Ministère des Affaires étrangères. De surcroît, la représentation de la RFY à Moscou par un ambassadeur, Borislav Milošević, qui n'est autre que le frère du Président yougoslave, conforte la perception dominante, dans les cercles du pouvoir russe, d'une Yougoslavie gouvernée "à la monégasque", par un clan familial qui contrôle la politique et l'économie ${ }^{15}$. S'ajoute à cela une connaissance assez approximative de l'armée yougoslave. En dépit des nombreuses coopérations passées et en cours (formation des officiers, fourniture de matériels), l'armée yougoslave, qui était hors du Pacte de Varsovie, a toujours entretenu avec les Soviétiques des rapports de méfiance réciproque. Enfin, pour conclure sur les motifs de cet "alignement" final, mentionnons la mauvaise évaluation, par la Russie, de l'articulation des diverses dimensions de la politique américaine, et notamment de la capacité des dirigeants des États-Unis à mobiliser un appareil médiatico-idéologique efficace et mobilisateur, à imposer leur argumentaire et, par ce biais, leur solution politique. Le grand professionnalisme des services de renseignement et de désinformation soviétiques, souligné par maints observateurs ${ }^{16}$, s'appuyait sur des institutions qui ont visiblement souffert des réformes politiques et économiques. Il apparaît que les leçons de la guerre du Golfe n'avaient pas été tirées chez les dirigeants russes. Les débats suscités au sein des sociétés civiles

14 Sur les troupes russes en Yougoslavie, Dervin (Sandrine), La Russie et le maintien de la paix en exYougoslavie, DEA Analyse comparative des aires politiques, IEP de Paris (dir. Anne de Tinguy), 2002. Cf. pour la période précédant l'intervention de l'OTAN au Kosovo Hoppe (Hans-Joachim), " Rußland und der Jugoslawienkonflikt ", Bericht des Bios, (14), 1997.

${ }^{15}$ En ce sens, une analyse du système politique yougoslave publiée dans l'hebdomadaire Kommersant Vlast', le 16/06/98.

${ }^{16}$ Cf.l'ouvrage de référence de Shultz (Richard), Godron (Roy), Dezinformatsia : mesures actives de la stratégie soviétique, Paris : Entrepôt, 1985. 
européennes par les guerres yougoslaves des années 1990 n'ont pas été attentivement suivis et analysés. Aussi la crise du Kosovo emporte-t-elle une conséquence indirecte : en 2000, la Russie se dote d'une doctrine de l'information, premier pas de la construction d'instruments plus efficaces dans la guerre de l'information.

\section{LE MYTHE DU « NÉO-PANSLAVISME »}

Ni réelle résurgence du panslavisme du XIX ${ }^{e ̀ m e}$ siècle ${ }^{17}$, ni idéologie politique vraiment articulée, le néo-panslavisme est l'une des composantes d'une idéologie anti-occidentale diffuse dans certains milieux intellectuels et politiques russes et yougoslaves, idéologie aux contours mal définis mais mobilisatrice, réactivée à la faveur de la guerre menée par les pays occidentaux contre la Yougoslavie. Les sources contemporaines de l'anti-occidentalisme russe sont variées (néo-eurasisme, néo-bolchevisme, néo-nationalisme-grandrusse, néo-messianisme orthodoxe) et alimentées par les soubresauts de l'histoire récente : défaite de l'URSS dans la guerre froide, déliquescence de l'État, apparition d'un Lumpenproletariat instruit issu de l'ancienne classe moyenne qui se sent trahie par les réformes économiques. Outre le parti communiste et le mouvement de Vladimir Jirinovski, une nébuleuse de groupuscules politiques développe des thèses convergentes. Parfois rapidement présentées comme des survivances du courant slavophile du XIX ${ }^{\text {ème }}$ siècle, les oppositions russe et yougoslave à l'Occident dans les années 1990 sont en réalité construites sur des interrogations face la situation internationale présente : pourquoi (pour qui ?) et dans quelles conditions l'URSS et de la Yougoslavie ont-elles été démantelées ? Pourquoi les nationalismes périphériques (croate, ukrainien) et les insurrections de minorités musulmanes (Albanais du Kosovo, Tchétchènes) bénéficient-ils dans les médias occidentaux d'un crédit plus favorable que les nationalismes russe et serbe ? L'anti-occidentalisme se nourrit d'argumentations d'ordre géopolitique ${ }^{18}$. En Russie, il trouve à se cristalliser à la faveur des événements du Kosovo. Pour ses tenants les plus extrémistes, l'intervention armée de l'OTAN manifeste la réalité d'un complot occidental. " Aujourd'hui Belgrade, demain Moscou ! " scandent les manifestants russes contre la guerre. Toutefois, loin des mouvements de masses sporadiques, l'anti-occidentalisme

${ }^{17}$ Sur ce point, Fišera (Vladimir Claude), Les peuples slaves et le communisme de Marx à Gorbatchev, Paris : Berg International, 1992.

${ }^{18} \mathrm{Cf}$. les écrits du chef de file du courant néo-eurasiste, Alexandre Douguine. Cf. Laruelle (Marlène), " Alexandre Dugin : esquisse d'un eurasisme d'extrēme-droite en Russie post-soviétique n, Revue d'études comparatives Est-Ouest, 32 (3), septembre 2001. 
- en Russie comme en Serbie - est d'abord un terrain intellectuel dont la seule tentative de synthèse théorique à ce jour est l'oeuvre de l'ancien dissident Alexandre Zinoviev ${ }^{19}$. Les écrits de l'ancien logicien de l'Académie des sciences de l'URSS, devenu professeur à la faculté de philosophie à l'Université de Moscou après son retour d'Allemagne, sont traduits en serbe et ont été souvent cités par l'épouse du Président Milošević, Mirjana Marković, à l'appui de ses discours.

Outre cette précaire internationale slave des intellectuels dits rougesbruns, les réseaux de volontaires russes qui combattent au côté des milices serbes en Croatie, en Bosnie et au Kosovo à partir de 1992 constituent la seule manifestation concrète du néo-panslavisme ${ }^{20}$. Au moment où interviennent les premières frappes de l'OTAN contre Belgrade, l'engagement de quelques centaines d'hommes - tout au plus 500 entre 1992 et 1999 - est présenté comme un phénomène de masse potentiel par certains médias russes. Dès le 24 mars, l'état-major du parti de Jirinovski annonce qu'il travaille à la mise en place de bureaux d'engagement volontaire pour la Yougoslavie sur l'ensemble du territoire de la Fédération de Russie et que des milliers de jeunes hommes répondent à l'appel et se tiennent prêts à partir en Yougoslavie " pour défendre leurs frères slaves contre l'invasion américaine $"^{21}$. Le 2 avril, la télévision publique annonce que 15000 volontaires ont déjà signé leur engagement à Moscou et 55000 à l'échelle de toute la Russie. Les coups de fil aux filières de recrutement se multiplient dans l'euphorie des manifestations de la jeunesse aux portes de l'ambassade et des consulats américains. Les passions se déchaînent dans la classe politique russe. En une semaine, la Yougoslavie devient l'exutoire de tous les échecs et de toutes les angoisses. Les leaders communistes et nationalistes, ainsi que plusieurs délégations de députés de la Douma de toutes obédiences politiques, y compris de la tendance dite réformatrice proche du pouvoir, se précipitent à Belgrade pour se faire prendre en photographie avec un T-shirt Target ou devant un pont détruit par les bombardements, en compagnie de Vojislav Šešelj, de Mirjana Marković ou de leurs homologues yougoslaves moins célèbres, avec lesquels ils conversent dans un mauvais anglais et trinquent bruyamment devant les caméras de la télévision ${ }^{22}$. L'enthousiasme des discours et des toasts prononcés à Belgrade gagnant la classe politique à

19 Zinoviev (Alexandre), L'occidentisme : essai sur le triomphe d'une idéologie, Paris : Plon, 1995. Une édition encore plus complète de ses thèses est parue à Moscou en 2000 : Na puti k sverkhobscestvu, éd. Centrpoligraf.

20 Signalons aussi l'existence de contacts établis entre les Églises orthodoxes serbe et russe. Sur ce point l'enquête de Babasjan (Natalija), "Sojuzniki ", Russkaja zurnal, 15/06/99 (http://www.russ.ru/politique/articlezo025).

${ }^{21}$ Cité par Russkaja Mysl', 29/03/99.

${ }^{22} \mathrm{Cf}$. le reportage du correspondant de l'hebdomadaire Itogi, 15/05/99. 
Moscou, une déclaration solennelle de quelques hommes politiques de premier plan est lancée en faveur de l'envoi d'une force militaire d'aide à la Yougoslavie. Cet appel est relayé par certaines personnalités officielles, dont le maire de Moscou, Iouri Loujkov, et l'un des ministres-adjoints de la Défense, qui doivent aussitôt se dédire, ayant oublié que, dès le 25 mars, le chef d'Etatmajor avait déclaré de manière appuyée que la Russie n'engagerait pas ses troupes dans le conflit... On ne peut que constater que la vague de néo-panslavisme soudain en vogue à Moscou obéit plutôt aux règles du spectacle qu'aux normes de la définition d'une politique en temps de guerre.

En dépit de leur grossièreté qui frise parfois l'indécence eu égard aux vraies victimes d'une vraie guerre, ces manifestations médiatiques vont être prises très au sérieux par certains analystes et commentateurs occidentaux. $\mathrm{Au}$ coeur du conflit, une expertise se fonde largement sur ces manifestations pour appuyer la thèse de l'imminence d'une nouvelle guerre froide. Un rapport publié sous l'égide d'un Centre de recherches de l'Académie britannique Sandhurst donne le ton : " bien qu'à l'heure actuelle la Russie préférera toute solution négociée même défavorable (...) nous sommes toujours en présence de deux conceptions fondamentalement différentes du monde : les Russes, une conception géopolitique ; les Occidentaux, une conception fondée sur le respect du droit international " et appelle à la vigilance des gouvernements occidentaux contre " l'activité intense des services secrets et des réseaux russes en Yougoslavie $"^{23}$. L'hypothèse d'une entrée en guerre de la Russie aux côtés de la Yougoslavie, même si elle est largement envisagée comme peu sérieuse, n'est jamais complètement exclue des scénarios et de l'arrière-plan des analyses. On retrouve de nombreuses citations du rapport Sherr-Main dans des éditoriaux de la grande presse anglophone au cours des mois de mai-juin 1999. Bien malgré eux, les auteurs de ce rapport d'expertise inspirent de nombreux développements médiatiques sur l'opposition ontologique entre les valeurs occidentales et l'" idéologie panslaviste néo-communiste ", développements qui préparent le terrain préalable d'une campagne de presse anti-russe qui commence en juin 1999, à l'heure où les grandes puissances négocient leur part d'influence au sein de la KFOR. Le quotidien britannique The Guardian relaie une information selon laquelle l'hypothèse d'une "participation massive de Russes " au " génocide des Albanais intervenu au Kosovo " est vérifiée. Quelques jours après la publication de cet article, Kenneth Bacon, le porte-parole du Pentagone, annonce que " nous savons avec certitude que des Russes ont participé aux massacres en série (...). Je ne dispose pas des vérifications nécessaires : a-t-on affaire à des unités ou des groupes, et de quelle taille ? Je suis profondément convaincu qu'il y a eu une participation russe". Bacon parle de

${ }^{23}$ Sherry (James), Main (Dr. Steven), Russian and Ukrainien Perceptions of Events, Sandhurst : The Conflit Research Centre, 25 avril 1999, 27 p. 
" participation russe ", et non plus de la " participation de Russes ", n'écartant donc pas l'hypothèse que " les Russes " en question aient été plus ou moins directement liés à " la Russie " en tant qu'État. Rapidement démenties, les informations relayées par The Guardian participent de l'amorce de campagne de désinformation dont l'origine demeure inconnue dont le néo-panslavisme constitue la toile de fond explicative ${ }^{24}$. Une fois de plus, on constate que la crise du Kosovo constitue le terrain d'une redoutable guerre de l'information dont toutes les batailles sont, hélas, loin d'être toutes soigneusement consignées dans des archives accessibles aux futurs historiens.

\section{DE LA DOCTRINE BREJNEV À LA DOCTRINE VIAKHIREV ?}

La crise du Kosovo met en évidence la dépendance énergétique de la Yougoslavie à l'égard de la Russie. La Yougoslavie n'occupe cependant pas une place décisive dans la stratégie énergétique russe en Europe balkanique, sachant que c'est la Bulgarie, par laquelle transitent tous les gazoducs, qui constitue le nœud gordien de la géopolitique régionale du géant Gazprom, le monopole gazier russe ${ }^{25}$. La dépendance yougoslave à l'égard des hydrocarbures russes - qui fournissaient en 2000 près de $30 \%$ de l'énergie consommée dans le pays - n'est pas tant un facteur géopolitique que proprement politique. Les réseaux soviéto-yougoslaves des ministères économiques établis pendant la période socialiste sont à l'évidence toujours actifs. A l'instar des nombreux réseaux économiques et politiques constitués à partir de la fin des années 1970 et entretenus par le géant gazier russe dans les pays européens, la connaissance que l'on en a aujourd'hui est à peu près presque nulle. Ces réseaux se sont perpétués et reconvertis. Le choix de l'ancien gazier Tchernomyrdine comme émissaire du gouvernement russe auprès des autorités de Belgrade vise probablement à rappeler aux autorités yougoslaves et au monde que si connivence russo-yougoslave il y a, celle-ci est d'abord marquée du double sceau de la dépendance de Belgrade et des intérêts économiques de Moscou. Viktor Tchernomyrdine entretient d'excellents rapports avec Mirko Marjanović, ancien Premier ministre serbe (dans les années 1990) et directeur de NIS-Progrestrade, une société qui détient le monopole de l'importation du

\footnotetext{
24 Sur cette affaire, Mjalo (Ksenija), op. cit., pp. 301-303.
}

25 Cf. un article de référence de Bonin (Peter), " Economical Levers in Russian Foreign Policy ", Paper for the 51st Political Studies Association Conference, 10-12 Avril 2001, Manchester, United Kingdom, 17 p. L'auteur est membre du Mannheim Center for European Social Research et prépare une thèse sur "la politique étrangère russe à l'égard de la Bulgarie et de la Yougoslavie dans les années 1990". 
gaz russe en Yougoslavie. Les rapports entre les gaziers russes et les importateurs yougoslaves remontent au moins au milieu des années 1970. Dans les années 1990, NIS-Progrestrade et Gazprom ont multiplié les accords de barter, ce qui a permis à Marjanović de contrôler indirectement l'activité de nombreuses branches économiques et d'occuper une position centrale dans l'économie yougoslave. Les gaziers russes et yougoslaves ont en commun d'être au coeur de toutes les transactions économiques et politiques de leurs pays respectifs. La Yougoslavie ayant accumulé une importante dette énergétique, Gazprom tente aujourd'hui (2001-2002) de convertir ces dettes en prises de participation dans des entreprises yougoslaves. C'est par ce biais que le géant gazier russe, devenu une holding en pleine internationalisation (il détient une part non négligeable du géant gazier allemand Ruhrgas), tisse sa toile en Europe balkanique.

Le 4 avril 1999, Vagit Alikperov, patron de la holding pétrolière russe Loukoïl, déclarait qu' " il ne faut pas oublier que les jets américains qui bombardent Belgrade sont approvisionnés par du pétrole russe ${ }^{26}$. L'exportation d'énergie devient le vecteur de l'influence russe en Europe. Un analyste bulgare - Ivan Krastev - a écrit que dans les années 1990, la doctrine Brejnev a été remplacée, par la doctrine Viakhirev (du nom du patron de Gazprom jusqu'en $2001)^{27}$.

Au-delà de l'étude du jeu diplomatique classique et des mythologies influant les perceptions mutuelles des divers belligérants, la crise du Kosovo, comme toutes les crises internationales contemporaines, invite à poursuivre l'analyse des relations internationales en Europe dans deux directions essentielles : mieux comprendre les guerres de l'information dans le contexte de l'après-guerre froide, mieux connaître les réseaux économiques Est-Ouest tissés pendant la période Brejnev - au moment où l'URSS construit ses gazoducs et oléoducs vers l'Europe et développe l'exportation à grande échelle de ses matières premières vers l'Europe - et leur pérennité. 